\title{
Construct Validation of the Health Belief Model
}

\author{
K. Michael Cummings, M.P.H. \\ Alan M. Jette, M.P.H. \\ Irwin M. Rosenstock, Ph.D.*
}

A multitrait-multimethod design was employed to assess the construct validity of the Health Belief Model. The data were obtained from a nonrepresentative sample of 85 graduate students at The University of Michigan's School of Public Health. The traits consisted of the respondents' perceptions of: health interest, locus of control, susceptibility to influenza, severity of influenza, benefits provided by a flu shot, and the barriers or costs associated with getting a flu shot. Each trait was measured by three methods: a sevenpoint Likert scale, a fixed-alternative multiple choice scale, and a vignette. The results indicate that the Health Belief Model variables can be measured with a substantial amount of convergent validity using Likert or multiple choice questionnaire items. With regard to discriminant validity, evidence suggests that subjects' perceptions of barriers and benefits are quite different from their perceptions of susceptibility and severity. Perceptions of susceptibility and severity are substantially but not entirely independent. Perceived benefits and barriers demonstrate a strong negative relationship which suggests the possibility that these two variables represent opposite ends of a single continuum and not separate health beliefs.

These preliminary results provide the basis for developing brief health belief scales that may be administered to samples of consumers and providers to assess educational needs. Such needs assessment, in turn, could then be used to tailor messages and programs to meet the particular needs of a client group.

The iterative nature of science requires a constant shifting of attention between concepts and their measures. Concepts with validated measures advance science; concepts without validated measures provide, at best, a way of viewing a domain. Measures, in the absence of articulated concepts, are empty. One of the formulations advanced over the past 20 years to explain individual health behaviors, the Health Belief Model, $^{2}$ has received

* K. Michael Cummings and Alan M. Jette are doctoral candidates in the Department of Health Behavior and Health Education, Irwin M. Rosenstock is Professor and Chairman of the Department of Health Behavior and Health Education, The University of Michigan, School of Public Healih.

Address requests for reprints to Mr. Cummings, Department of Health Behavior and Health Education, The University of Michigan, School of Public Health, Ann Arbor, Mfichigan 48109. 
considerable attention and study, but has not been the subject of intensive psychometric development. Much more attention has been given to developing the concepts used in this model, than to the validity of measures of those concepts. The model, however, has yielded substantial evidence of utility in predicting health actions despite this deficiency. The present study attempted to demonstrate empirically the construct validity of the Health Belief Model (HBM) variables using the multitrait-multimethod scheme described by Campbell and Fiske. ${ }^{4}$

The HBM hypothesizes that a decision to undertake a health action will not be made unless the individual is psychologically ready to take action relative to a particular health threat or condition. Readiness to act is defined by the extent to which:

1. the individual feels susceptible to the condition in question and the extent to which its possible occurrence is viewed as having serious consequences;

2. the individual believes that there are actions which would be beneficial in reducing his/her susceptibility to and/or severity of the condition should it occur; and

3. the individual believes that the psychological costs associated with taking the health action are outweighed by the benefits to be derived.

The original formulation was oriented solely toward the desire to avoid a specific disease threat; this approach has been reformulated and expanded to include a dimension of general health motivation. ${ }^{3}$ The health motivation dimension was added to represent relatively non-specific and stable differences in individuals' health interest as well as perceptions of control over health matters.

Reviews of research using the HBM provide substantial evidence of the model's utility in predicting health actions. The reliability of HBM measures has been studied, ${ }^{9}$ but little is known about the validity of the techniques used to measure its component beliefs. In fact, many studies using the theoretical perspective of the HBM have used quite different questions intended to measure the presence and magnitude of the same health beliefs. Although one might argue that the demonstrated utility of the model, even when different measures are used, argues for its validity, this approach risks serious problems; for example, as the measures change from study to study, the constructs being measured may also vary. Consequently, in the absence of a demonstration of the ability to measure validly the constructs included in the HBM, the practical utility of the formulation is reduced. This is especially true if one wishes to identify and modify specific beliefs as a means of influencing behavior.

This pilot study has both substantive and methodological aims:

1. to demonstrate the degree to which some of the current measurement techniques validly measure the health beliefs they are purported to measure;

2. to assess the degree of independence among the various components of the HBM; and

3. to compare the advantages of the Campbell-Fiske approach to estimating 
construct validity with a method of structural equation analysis.

\section{METHODS}

\section{Population}

The data were collected from a non-representative sample of 85 graduate students at the University of Michigan's School of Public Health registered in a Master of Public Health survey course. Fifty-one percent of the sample were female. The respondents ranged from 22 to 45 years of age with $78 \%$ under 30 years of age.

\section{Measures}

A written questionnaire was constructed which attempted to measure six hypothetical constructs or traits by each of three separate methods.

The traits consisted of respondents' self-report of perceptions of:

HI-interest in health marters,

LC-locus of control over health matters,

SUS-susceptibility to influenza,

SEV-severity of influenza,

BENE-the benefits provided by a flu shot, and

BAR - the barriers/costs of receiving a flu shot.

Each trait was measured by each of three methods:

1. Respondents rated the direction and degree of intensity of their attitude toward a particular trait on a seven-point Likert scale. For example:

Many times I feel that I have little control over my health-that is, where would you place yourself on this scale. (Circle one number.)

$\begin{array}{cc}\text { Strongly } & \text { Strongly } \\ \text { Agree } & \text { Disagree }\end{array}$

$\begin{array}{lllllll}1 & 2 & 3 & 4 & 5 & 6 & 7\end{array}$

2. Presented with a fixed number of labeled alternatives, respondents were instructed to choose one item which best represented their feelings toward a specific trait. For example:

Suppose you were to get the flu in the next 12 months. How serious do you think this condition would be for you? Would you say it would be..... (place a check mark $[\checkmark]$ next to your response).

( ) extremely serious

( ) quite serious

( ) somewhat serious

( ) not at all serious

3. Respondents were presented with a pair of vignettes describing two individuals and their attitudes toward a trait. Persons rated the extent to 
which they saw themselves like one or the other of these persons on a fivepoint scale. For example:

Please read what Alice is like and what Sue is like. Then indicate the extent to which you are like either Alice or Sue.

Alice worries a lot about her health. Recently, she missed several days of work because of the flu. In fact, she still has a bad cough and little energy because of her sickness. It seems every time Alice gets the flu, it is a bad case.

Sue also is concerned about her health. Unlike Alice, however, whenever Sue becomes ill it is only for a little while, and is usually not very serious. For instance, when Sue got the flu a week or so ago, she was up and around within a day and back to work.

Who are you like? (Circle one number.)

\begin{tabular}{|c|c|c|c|c|}
\hline $\begin{array}{c}\text { A lot } \\
\text { like Alice }\end{array}$ & $\begin{array}{l}\text { Somewhat } \\
\text { like Alice }\end{array}$ & $\begin{array}{l}\text { Halfway } \\
\text { between }\end{array}$ & $\begin{array}{l}\text { Somewhat } \\
\text { like Sue }\end{array}$ & $\begin{array}{c}\text { A lot } \\
\text { like Sue }\end{array}$ \\
\hline 1 & 2 & 3 & 4 & 5 \\
\hline
\end{tabular}

Analysis

An investigation of construct validity depends on both: (a) a network of relationships among a set of observed measures, and (b) a series of theoretical assumptions about the relationships of a set of hypothetical constructs to one another and to the observed measure. ${ }^{1}$ Few attempts have been made to obtain direct estimates of construct validity.

Campbell and Fiske argued that evidence for construct validity exists when there is convergence between independent measures of the same trait and discrimination between measures of different traits. They showed that it is possible to examine convergence and divergence within a matrix of intercorrelations of two or more theoretically unrelated traits measured by two or more independent methods. Evidence for convergence exists when the correlations between measures of the same trait are positive and significantly different than zero (validity diagonal entries in Table 1).

Evidence for discrimination is three-fold. First, one should compare the correlations in the validity diagonal with those values in the same column and row in which neither trait nor method are in common (different trait-different method triangles in Table 1). The observed correlations between measures of the same trait using different methods should be greater than the correlations between measures of different traits using the same method. In this comparison, the difference among correlations between measures of the same traits (which should be high) and correlations between measures of different traits (which should be low if the underlying traits are different) are examined.

Second, the correlations in the validity diagonal should be greater than 


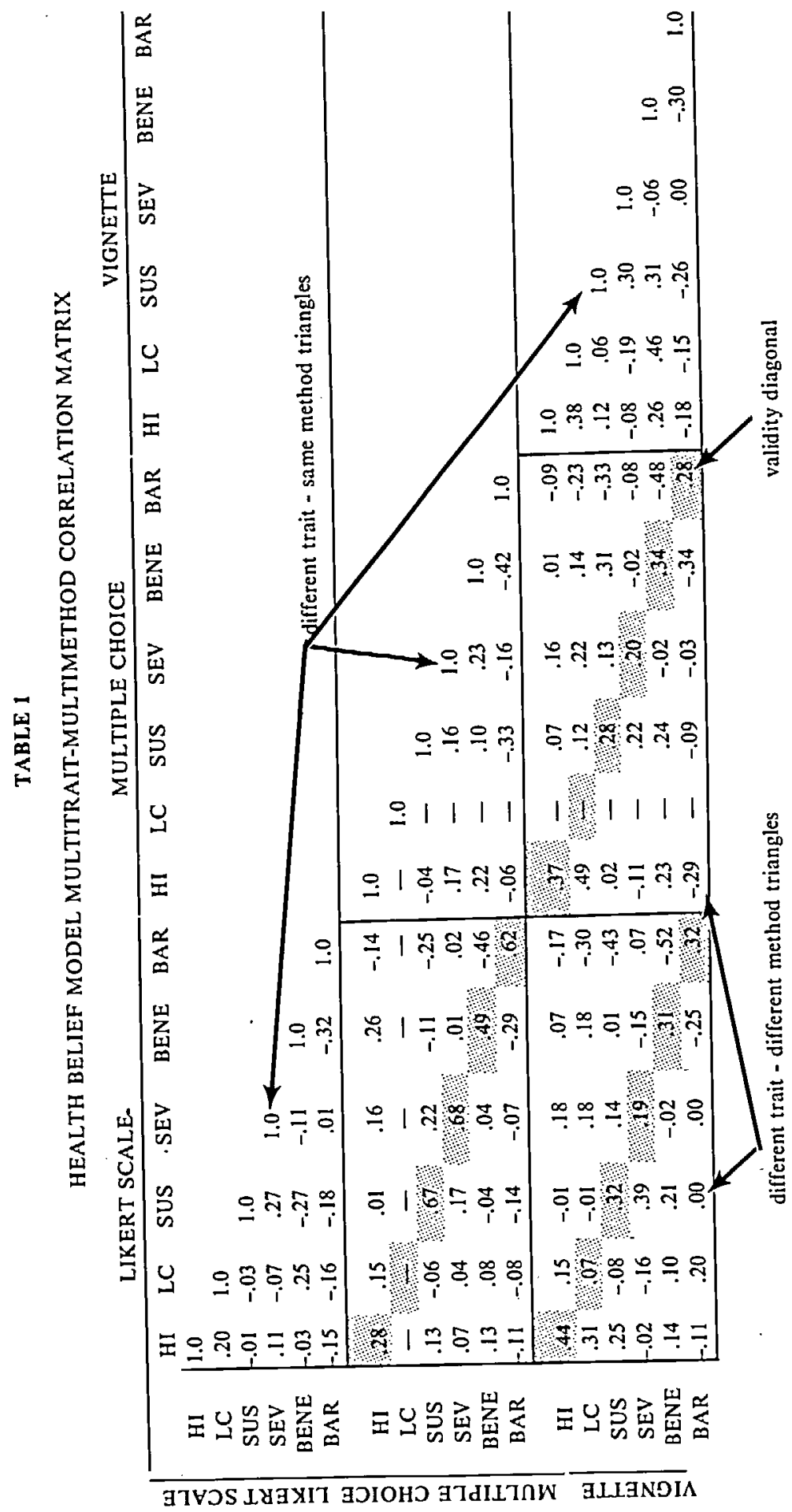


those correlations between the same trait and other traits with a common method (different trait-same method triangles in Table 1). In other words, different traits measured by the same method should have a lower correlation than different measures of the same trait. Otherwise, high correlation between different traits using the same method may be attributed to the measurement procedure.

Third, the pattern of trait interrelationships should be the same within and between different measures. A similar pattern of correlation among items is evidence of an underlying set of true relationships among traits which is maintained in spite of possible method effects (due to either a spurious correlation because the same method was used, or lack of independence between different methods).

Although the Campbell and Fiske approach represented an important advance at the time, it is unable to provide precise estimates of construct validity, and in the case of large matrices is quite awkward to interpret. A number of methods for quantifying the multitrait-multimethod approach have been made in the psychological literature. ${ }^{5,8}$ One of the most promising new techniques for assessing construct validity is structural equation analysis.

Jöreskog ${ }^{6,7}$ developed a powerful maximum likelihood technique which partitions the variance of a measure into three portions; valid variance (reflecting what the measure is intended to measure); correlated error variance (reflecting influences other than those the measure was designed to tap which also affect other measures); and residual variance (variance which is not otherwise accounted for).

The general form of the structural equation model used in this investigation appears in Figure 1. The variance of the observed measures are represented by the rectangles. The circles on the left are linked directly to all the measures intended to tap an identical trait, but not to measures intended to tap other traits. This helps to determine the meaning of the circles on the left-i.e., they represent what the observed measures have in common, which in this case is the respondent's feelings about a particular health belief. The linkages between traits incorporates an expectation that some of the health beliefs will be related.

A similar set of assumptions are involved in the specification of linkages with the circles on the right. These linkages are intended to represent method effects (correlated error variance). In addition to the pattern linkages, a constraint was imposed on the model with regard to the linkages between the circles on the right (method factors) and the rectangles (measures). The magnitude (whatever it might be) of the linkages from any one method factor was constrained to be equal for all measures using the same method.

Parameter estimates (regression coefficients) of the linkages between the observed measures and traits (construct validity coefficients) were obtained by application of the LISREL computer program. ${ }^{6}$ The square of the validity coefficient indicates the proportion of a measure's variance which is valid. Similarly, the square of the method effect coefficient indicates the correlated 
FIGURE 1.

GENERAL FORM OF STRUCTURAL EQUATION MODEL AND PARAMETER ESTIMATES FOR A SUBSET OF THE TRAITS

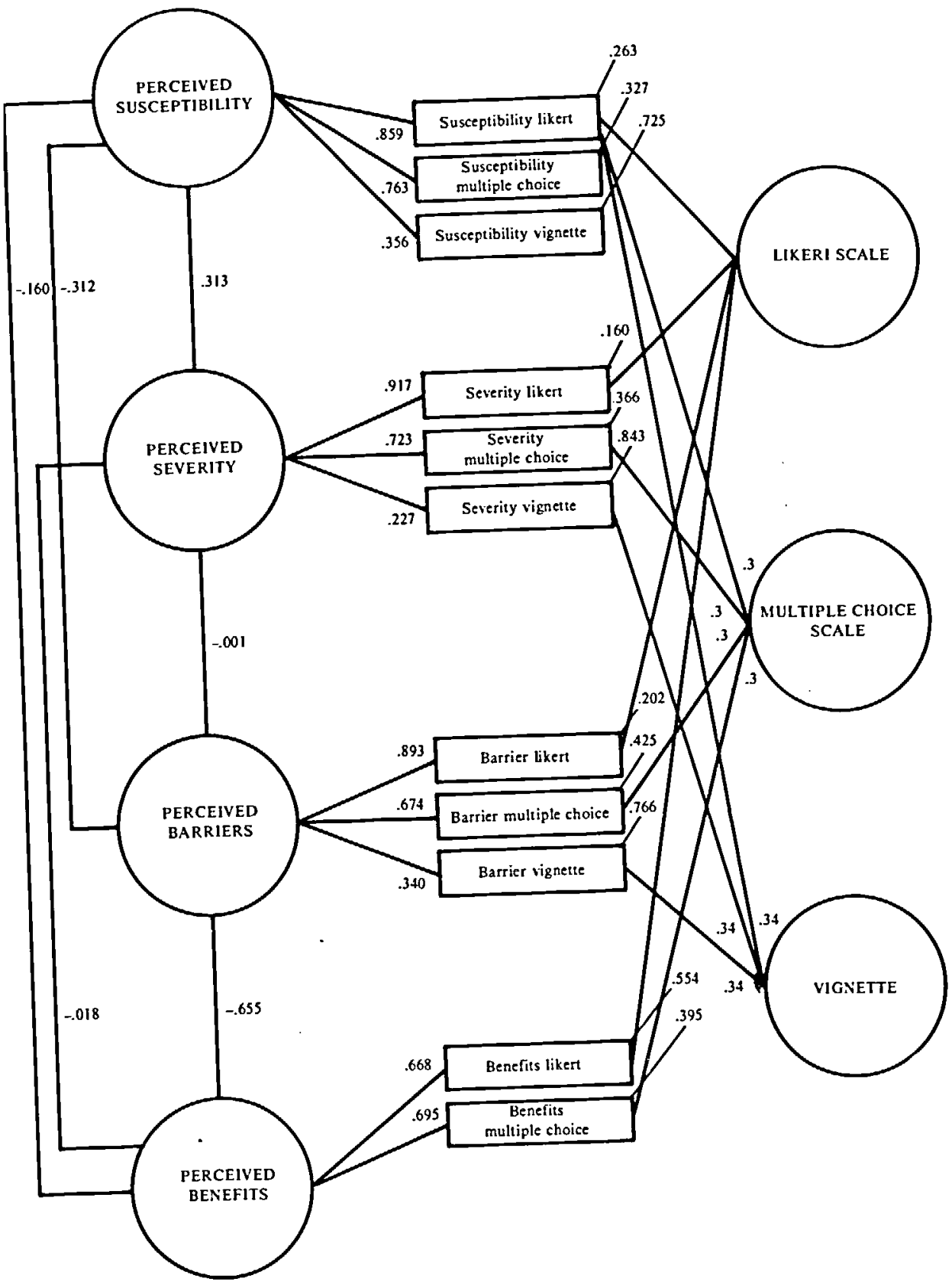


error, or bias of the measure's variance.*

\section{RESULTS}

\section{Multitrait-Multimethod Approach}

The multitrait-multimethod correlations (Pearson r's) are presented in Table 1. The highest correlations are found in the validity diagonals, suggesting a substantial degree of convergence. This finding is true for all of the traits except locus of control which exhibits only a weak association (.07) between measures. The greatest degree of convergence is seen between the Likert and multiple choice scales.

Comparison of the correlations in the validity diagonals with those in the different trait-different method triangles indicate low discriminability between barriers and susceptibility, barriers and benefits, susceptibility and severity, and locus of control and health interest. A similar result is obtained when the values in the validity diagonals are compared to correlations between that trait and other traits with a common measure (different traitsame method triangle). Moreover, the vignette measures show less ability to discriminate among traits than either the multiple choice or Likert scale methods. Finally, a consistent pattern of trait correlations is found within and between different measures.

In summary, evidence for convergence is obtained for five out of the six health belief traits. Discrimination among traits is less clear cut. Substantial correlations are found between different and same measures of barriers and susceptibility, barriers and benefits, susceptibility and severity, and locus of control and health interest. Among the measures, the Likert scale exhibits the best discrimination among traits, followed by the multiple choice and vignette methods.

\section{Structural Equation Analysis}

The results of the structural equation analysis are represented by the parameter estimates included in Figure 1. As shown in Figure 1, the three methods-Likert scale, multiple choice scale, and vignette-produced data with an average validity coefficient of 0.64 . Data obtained using the vignette method had substantially lower average validity $(0.30)$. The Likert scale and multiple choice scale showed average validities of 0.82 and 0.71 respectively. On the basis of these results, one can infer that single item measures using either the Likert or multiple choice scales to assess an individual's beliefs about susceptibility to flu, severity of flu, benefits from a flu shot, and barriers to obtaining a flu shot, contain approximately $60 \%$ valid variance. Assessing the same beliefs through the vignette method results in only about $9 \%$ valid variance.

*For a description of how coefficients are derived in the measurement model see Jöreskog. K.G. and Sorbom, D. "LISREL III: Estimation of Linear Structural Equations Systems by Maximum Likelihood Methods." User's Guide, 1976. National Educational Resources, Inc., P.O. Box A3650, Chicago, Illinois 60690 . 
The magnitudes of the method effect coefficients indicate that for the multiple choice and vignette methods, roughly $10 \%$ of the total variance could be attributed to method effects. The Likert scale showed zero method effects. This unreasonable method effect for the Likert scale can probably be attributed to unstable correlation coefficients resulting from the small sample of data available.

The residual variance for each of the observed measures are shown in Figure 1. The results indicate a substantial amount of unaccounted for variance (approximately 45\%) for each of the observed measures. The observed measures which include the vignette method have the largest proportion of uncorrelated error variance $(78 \%)$, followed by the multiple choice $(38 \%)$ and Likert scale $(29 \%)$ methods.

One other set of parameter estimates appears in Figure 1. The linkages between traits indicate a strong negative relationship between barriers and susceptibility (-.312), barriers and benefits $(-.655)$, and a positive relationship between susceptibility and severity (.313).

The model shown in Figure 1 represented a reasonable fit to the data, with the estimated correlations (of which there are 66) among the measures deviating only slightly from the observed measures (mean deviation of 0.06 ).

\section{DISCUSSION}

Conclusions based on the analysis of convergent validity are clear. The original components of the HBM-perceptions of susceptibility, severity, barriers and benefits-can be measured with substantial convergent validity using questionnaire or interview items. However, some methods of measurement appear to be better than others. The seven-point Likert scale and the multiple choice method both converge on the HBM variables, with the Likert scale showing some superiority. The vignette approach demonstrates poor convergence. Further evidence of the shortcomings of the vignette method is revealed by its singularly high residual variance (78\%). On the basis of these findings, vignette questionnaire items are not recommended for measuring health beliefs.

With respect to discriminant validity, the evidence is that the perceptions of barriers and benefits are substantially different from those of perceptions of susceptibility and severity. However, the moderate negative relationship between perceived susceptibility and perceived barriers was not anticipated (-.312). It may reflect an inability to adequately discriminate between these constructs with the methods used in this study; or, it may reflect an effort at achieving cognitive balance. The person who perceives imposing barriers to taking an action may begin to believe that the action is not required in his case. The present data do not permit a resolution of this issue.

The moderate positive correlation $(.313)$ between perceived susceptibility and perceived severity suggests that while these are different concepts; they overlap somewhat. This modest positive correlation was anticipated based on past theoretical and empirical work where perceptions of susceptibility and 
severity have been hypothesized to represent an individual's perceived threat to a particular disease. ${ }^{9}$

The substantial negative correlation (-.655) between perceived barriers and benefits should also be noted. This finding suggests that as one's perception of the degree of benefits from taking a certain health action increases, one's perception of the barriers to the same health action concomitantly decreases. This suggests that perceived barriers and benefits may represent opposite ends of a single continuum and should not be treated as separate health beliefs.

The magnitude of the relationships among the various health beliefs can also be observed by inspecting the correlations in the multitrait-multimethod mat rix using the criteria recommended by Campbell and Fiske. However, the difficulties involved in attempting this task, even on this relatively small matrix, illustrate one of the many advantages of the structural modeling approach. An overall comparison of the two approaches to estimating the degree of construct validity show that both procedures lead to similar conclusions.

The structural equation approach has the advantage of providing the researcher with precise, easily interpretable quantitative estimates of "a measure's valid, correlated, and residual variance. However, the apparent convenience of interpreting structural equation coefficients tends to camouflage some possible underlying problems with the structural modeling approach. For instance, to estimate the parameters in structural equation models, the investigator makes the following assumptions:

1. the causal relationships between variables have been correctly specified;

2. the errors of measurement are uncorrelated, and have a constant variance;

3. the observed variables have a multivariate normal distribution; and

4. the relationship among variables is assumed to follow a linear, additive system.

The specified effects of violating any of these assumptions on the structural equation solutions are difficult to assess given the relative newness of the technique. Although Jöreskog and others ${ }^{7}$ have proposed ways of dealing with non-linearity, interactions, multicollinearity, and non-recursiveness, the procedures for handling these problems are at present debatable. In summary, caution should be employed in recommending the structural equation approach over the more established Campbell and Fiske multitraitmiltimethod procedure without taking account of the specific assumptions underlying both techniques.

The reader's attention is once again drawn to the non-representativeness of the present data, both with respect to sampling and to content. The sample of public health students is clearly not representative of any general population, the content was limited to influenza, and only three methods were used. It is quite possible that these results would not be replicated using different health conditions and a different sample. However, since, in overview, external validity can never be deduced, replication is always essential, using both the 
same materials reported here with other samples, and using other content and other methods. The present results should aid in the iterative, scientific process described above. It is in that spirit that these tentative findings are provided.

\section{IMPLICATIONS}

These preliminary results, if replicated, could be of considerable practical importance. Perceptions of susceptibility to and severity of particular conditions as well as the perceived benefits/barriers associated with interventions have been shown to be different concepts, and may be validly measured using Likert-type and multiple choice questionnaire items. This provides a basis for developing brief health belief scales that may be administered to samples of consumers and providers to assess educational needs. Such needs assessment, in turn, could then be used to tailor messages and programs to meet the particular needs of a client group.

Health educators require pinpointed, tailored approaches to meet the educational needs of their clientele. However, methods of assessing educational need that would permit development of tailored messages for different individuals or sub-groups in a population have not been available. The Healt $h$ Belief Model suggests a means for solving at least part of this problem. If it is known that most consumers in a particular sub-group of interest do not believe they or their children are susceptible to a particular condition, that its occurrence would be serious, or that effective methods at acceptable costs exist for prevention or control, then more specific educational objectives can be set.

Until the present study was undertaken, however, there could be little confidence that any set of items would validly measure the concepts in the HBM. Now, within the limitations of this study, achievement of the objective of a standard set of questions that may be used to assess the concepts with reasonable validity is closer. With further replication of this work, extended to other samples and to other health conditions, it should be possible to accomplish this objective.

A final word of caution is required. Knowledge of HBM components does not constitute all that should be known, but rather some of what should be known in assessing educational needs. Secondly, the results of any needs assessment do not imply a best method for satisfying those needs. The Health Belief Model does not presuppose or imply any specific strategy for change. Some practitioners may attempt to modify beliefs by direct persuasive efforts; others may wish to modify the structure of the medical care system in order to change beliefs. In short, knowledge of the nature of a problem does not imply an ideal solution, but it is a necessary step in developing a rational and effective solution.

\section{ACKNOWLEDGEMENTS}

The authors wish to thank Frank Andrews for his helpful comments on data analysis and David Hunsche for preparation of the illustration used in the paper. 


\section{REFERENCES}

1. Andrews F, Crandall R: The validity of measures of sclf-reported well-being. Soc Indicators Res 3:1-19, 1967.

2. Becker $M$ (ed): The Health Belief Model and personal health behavior. Health Educ Monogr 2:236, 1974.

3. Hecker M, Maiman L: Sociobehavioral deteminants of compliance with health and medical care recommendations. Med Care 13:10-24, 1975.

4. Campbell DT, Fiske DW: Convergent and discriminant validation by the multitraitmultimethod matrix. Psychol Bull 56:81-105, 1959.

5. Jackson ON: Multimethod factor analysis in the evaluation of convergent and discriminant validity. Psychol Bull 29:259-271, 1969.

6. Jöreskog KG, van Thillo M: LISREL: A General Computer Program for Estimating a Linear Structural Equation System Involving Multiple Indicators of Unmeasured Variables. Res Bull, RB-72-56. Princeton, New Jerscy, Educational Testing Service, December 1972.

7. Jöreskog KG: A General Method for Estimating a Linear Structural Equation System, in Goldberger AS, Duncan OD (eds): Structural Equation Models in the Social Sciences. New York, Seminar Press, 1973.

8. Kavanage MJ, MacKinney AC, Wolins L: Issues in managerial performance; Multitraitmultimethod analysis of ratings. Psychol Bull 75:34-39, 1971.

9. Maiman L, Becker M, Kirscht JP, et al: Scales for measuring health belief model dimensions: A test of predictive value, internal consistency, and relationships among beliefs. Health Educ Monogr 5(3):215-230, 1977. 